\title{
ON A FORMULATION OF A NONLINEAR THEORY OF PLATES AND SHELLS WITH APPLICATIONS
}

\author{
Kamal A. Meroueh \\ Department of Aerospace Engineering, The University of Michigan, Ann Arbor, MI 48109, U.S.A.
}

(Received 3 October 1985)

\begin{abstract}
A nonlinear theory of plates and shells based on only one consistent kinematical approximation is employed to investigate finite strain and large deformation structural problems. The kinematics, expanded with respect to the thickness parameter, include consistently and adequately higher-order terms to allow fibers initially straight to deform nonlinearly in the large deformation range. A full Lagrangean formulation is considered. Starting from a three-dimensional principle of virtual work, the equilibrium equations and the resultant stresses of the shell together with their incremental form are derived employing an incremental constitutive relation valid for a wide class of rate-independent material. And, to assess the accuracy of the theory adopted, some boundary value problems are solved using a proposed hyperelastic material to model the constitutive behavior of a semi-infinite plate. The analytical and numerical (finite element method) results show good agreements between the exact and the approximate theories.
\end{abstract}

\section{INTRODUCTION}

Finite strain and large deformation problems are studied employing a theory of plates and shelis based on only one consistent kinematical approximation. Of particular interest are applications, such as in metal forming, which often result in large curvatures and significant shear effects. Higher-order terms in the displacement quantities are thus included in the formulation to properly account for realistic nonlinear behavior for thin and thick solids.

Based on a full Lagrangean description, an incrementally linear constitutive relation, well suited for the finite element method, is presented. This constitutive law is valid for a broad class of nonlinear materials such as: hyperelastic, hypoelastic as well as rate-independent elastic-plastic materials. In particular, a specific hyperelastic material function satisfying the growth and the ellipticity conditions is proposed and used in the numerical applications. In these cases the higher-order terms in the kinematics become significant especially when large strains and large deformations are considered.

Aside from the investigations developed using the Kirchhoff-Love [1] and Mindlin-Reissner [2, 3] approximations very few attempts were made to analyse nonlinear boundary value problems employing shell theories which include adequately in-plane and outof-plane deformation effects. In the context of small strain, it is worth mentioning the contributions of Sanders [4], Koiter [5] and Budiansky [6], who have promoted nonlinear theory of elastic shells. Whereas in the general field of nonlinear plate and shell theories in which the displacements are expanded into power series with respect to the thickness parameter, we note the works of Naghdi [7], Yokoo and Matsunaga [8] and Antman [9]. And for accounts of various theories of shells the reader is referred to the work of Naghdi [ 7$]$ and the associated references.

In spite of the progress in the derivation of nonlinear theories of plates and shells, very little effort has been focused on solving nonlinear boundary value problems. However, certain efforts on the numerical sides are noteworthy. Indeed, a large number of papers have been guided towards developing the so-called "degenerate" finite elements for nonlinear shell analyses (see Refs [10] and [11]).

\section{Scope}

This paper starts with the introduction of the kinematics. The equilibrium equations and the stress resultants are then derived from the full Lagrangean three-dimensional virtual work principle using the general form of the kinematical assumption adopted in this work. As to the stress-strain relation, a constitutive equation valid for a broad class of incrementally linear materials is employed. Also, a strain energy density function for rubber-like materials is proposed. The hyperelastic material exhibits quite good behavior even at fairly large strains.

To illustrate the effectiveness of the theory various sample solutions are presented. And in particular a finite strain pure bending problem for a semi-infinite plate is investigated analytically. Numerically, the finite element method is employed to compare the exact and the approximate theory for a plate subjected to a transversely distributed load and a shear load respectively.

The results show good agreement between the exact and the shell theory. Two higher-order cases are considered, namely displacement models in which terms up to second order and then up to third order of the thickness parameter are included. It is observed 


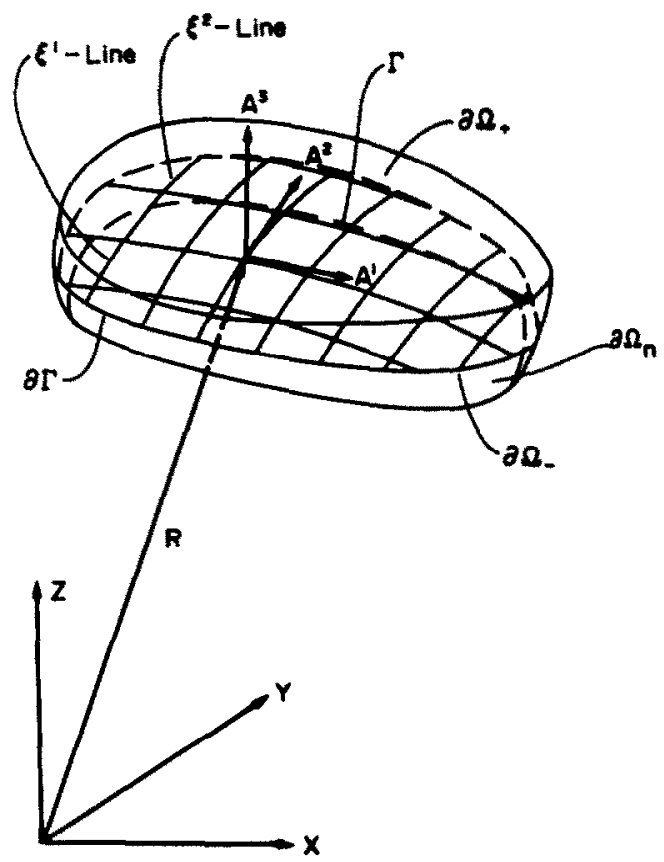

Fig. 1. Shell body in the undeformed configuration.

that the increase of one additional term in the kinematics leads to a fast convergency.

\section{DERIVATION OF THE TWO-DIMENSIONAL SHELL THEORY}

Within the context of finite continuum mechanics and using a Lagrangean approach, a nonlinear shell theory is presented based on a consistent kinematical assumption. The theory is valid for shells of arbitrary geometry and for a broad class of constitutive laws. The two-dimensional shell equations are to be consistently derived from the principle of virtual work of a three-dimensional continuum using a consistent higher-order kinematical assumption, thus avoiding the contradictions present in classical shell theories.

\section{Theory}

Consider a shell for which the convected coordinates $\left[\xi^{1}, \xi^{2}\right] \in \Gamma$ and $\xi^{3} \in\left[h_{1}, h_{2}\right]$ such that $\Gamma$ represents the reference surface of the shell and $\xi^{3}$ denotes the co-ordinate in the thickness direction bounded by the shell upper surface $\Omega_{+}$and lower surface $\boldsymbol{\Omega}_{-}$, let also $\boldsymbol{\Omega}_{n}$ denote the lateral surface boundary of the shell (see Fig. 1).

Assuming that the current position vector $p$, the reference position vector $\mathbf{P}$ and the displacement of a material point $\mathbf{O}$ to be adequately smooth function of their arguments, we adopt the following kinematical approximationt:

+ Upper case letters are utilized to denote quantities in the reference configuration and the lower case ones for the current configuration.

$$
\begin{aligned}
& \mathbf{p}=\sum_{r=0}^{R} f_{r}\left(\xi^{3}\right) \mathbf{d},\left(\xi^{\alpha}, t\right) \\
& \mathbf{P}=\sum_{r=0}^{R} f_{r}\left(\xi^{3}\right) \mathbf{D}_{r}\left(\xi^{\alpha}\right) \\
& \mathbf{U}=\sum_{r=0}^{R} f_{r}\left(\xi^{3}\right) \mathbf{U}_{r}\left(\xi^{\alpha}, t\right),
\end{aligned}
$$

where the integer $R \geq 2$ and $f_{r}$ are the Legendre polynomials in $\left[h_{1}, h_{2}\right]$. Also noting that $f_{0}=1$ we define the following surface quantities at $\xi^{3}=0$

$$
\mathbf{D}_{0}=\mathbf{R} \quad \mathbf{d}_{0}=\mathbf{r} \quad \mathrm{U}_{0}=\mathrm{U}
$$

which represent the reference position vector, the current position vector and the displacement vector of an arbitrary point in $\Gamma$, respectively. The vectors $\mathbf{d}_{r}, D_{\text {, are sometimes called in the literature director }}$ vectors for the shell (see, for example, Naghdi [7]).

From eqns (2.1) and (2.2) one may form the covariant basis vectors $\mathbf{g}_{i}$ and $\mathbf{G}_{i}$ in the current and reference states respectively

$$
\mathbf{G}_{i}=\partial \mathbf{p} / \partial \xi_{i} \quad \mathbf{G}_{i}=\partial \mathbf{P} / \partial \xi_{i}
$$

A deformed $d \omega$ and an undeformed $d \Omega$ volume element in the three-dimensional space are given by

$$
\begin{array}{ll}
\mathrm{d} \omega=g^{1 / 2} \mathrm{~d} \xi^{1} \mathrm{~d} \xi^{2} \mathrm{~d} \xi^{3} ; & g^{1 / 2}=\left(g_{1} \times g_{2} \cdot g_{3}\right) \\
\mathrm{d} \Omega=\mathbf{G}^{1 / 2} \mathrm{~d} \xi^{1} \mathrm{~d} \xi^{2} \mathrm{~d} \xi^{3} ; & G^{1 / 2}=\left(G_{1} \times G_{2} \cdot G_{3}\right) .
\end{array}
$$

At this point and without loss of generality it is also assumed that

$$
g^{1 / 2}>0 \quad G^{1 / 2}>0
$$

the above conditions ensure the physically motivated requirement that the mappings $\left(\xi^{1}, \xi^{2}, \xi^{3} ; t\right) \rightarrow \mathrm{p}$ are everywhere one to one and orientation preserving.

The volume change of a material element from the reference to the current configuration is given b;

$$
J=\mathrm{d} \omega / \mathrm{d} \Omega=\sqrt{ }(g / G),
$$

where $J$ is the Jacobian determinant of the transformation $\mathbf{P} \rightarrow \mathbf{p}$.

To this end some preliminary kinematics for the undeformed and deformed reference surfaces will be established in accordance to eqns (2.1) and (2.2).

The reference surface covariant base vectors are, in the current and reference configurations respectively, given by

$$
\mathbf{a}_{\alpha}=\partial r / \partial \xi^{\alpha} \quad \mathbf{A}_{\alpha}=\partial \mathbf{R} / \partial \xi^{\alpha} .
$$

The normal current and reference base vectors are easily found to be 


$$
a^{3}=a_{1} \times a_{2} \quad A_{3}=A^{3}=\left(A_{1} \times A_{2}\right) /\left|A_{1} \times A_{2}\right|,
$$

where $A_{3}\left(A^{3}\right)$ is normalized. The metric tensors of the deformed $\left(a_{\alpha \beta}\right)$ and undeformed $\left(A_{\alpha \beta}\right)$ surfaces at $\xi=0$ together with their respective inverses are

$$
\begin{aligned}
& a_{\alpha} \cdot a_{\beta}=a_{\alpha \beta} \quad a^{\alpha} \cdot a^{\beta}=a^{\alpha \beta} \\
& \mathrm{A}_{\alpha} \cdot \mathrm{A}_{\beta}=A_{\alpha \beta} \quad \mathrm{A}^{\alpha} \cdot \mathrm{A}^{\beta}=A^{\alpha \beta} .
\end{aligned}
$$

For later reference, we record the following important relations:

$$
\begin{aligned}
\mathbf{A}_{3, \alpha} \cdot \mathbf{A}_{\beta} & =-\mathscr{P}_{\alpha \beta} \\
\mathscr{H} & =\frac{1}{2} \mathscr{P}_{\alpha}^{\alpha}=\frac{1}{2}\left(\mathscr{D} \mid+\mathscr{O}_{2}^{2}\right) \\
\mathscr{H} & =\mathscr{M}_{1} \mid \mathscr{O}_{2}^{2}-\mathscr{D}_{2}^{2} \mathscr{W}_{1}^{2},
\end{aligned}
$$

where $\mathscr{B}_{\alpha \beta}$ is the second fundamental form of the reference surface and $\mathscr{H}$ and $\mathscr{H}$ in eqn (2.12) are two invariants: the mean curvature and the Gaussian curvature respectively.

The complete set of the reference surface kinematics requires the determination of the Christoffel symbols (employed in covariant differentiation), which can be defined using the metric tensors given in eqn (2.10). For a detailed discussion one may refer to the textbook of Green and Zerna [12].

In the reference state, and without loss of generality, one can conveniently parametrize the shell in a way that the position vector $P$ in eqn (2.1) in the initial configuration can be written as $\dagger$

$$
\mathbf{P}=\mathbf{R}+\xi \mathbf{D}_{1},
$$

where $D_{1}=A_{3}$ and $A_{3}$ is the basis vector normal to the surface $\Gamma$. From eqns (2.3), $(2.8)-(2.13)$, and taking into account that $D_{1}=A_{3}$ the following relations are derived:

$$
\begin{aligned}
\mathbf{G}_{\alpha} & =\left[\delta_{\alpha}^{\beta}-\xi \mathscr{O}_{\alpha}^{\beta}\right] \mathbf{A}_{\beta}=\mu_{\alpha}^{\beta} \mathbf{A}_{\beta} \\
\mathbf{G}_{3} & =\mathbf{A}_{3}=\mathbf{G}^{3}=\mathbf{A}^{3} \\
\mathbf{G}_{\alpha \beta} & =\mu_{\alpha}^{\gamma} \mu_{\beta}^{\delta} A_{y \delta} \\
\mathbf{G}^{\alpha \beta} & =\mu_{\gamma}^{\alpha} \mu_{\delta}^{\beta} A^{\gamma \delta} \\
\mu & =\operatorname{det}\left(\mu_{\alpha}^{\beta}\right)=\sqrt{ }(G / A)=1-2 \mathscr{H} \xi+(\xi)^{2} \mathscr{O},
\end{aligned}
$$

in which $\mathscr{H}$ and $\mathscr{H}$ are defined in eqn (2.12) $\left(\mu_{\alpha}^{\beta}\right.$ is often called "shifter" or "translator").

An important relation for the reference volume element $d \Omega$ can be established from eqns (2.5) and (2.14), namely,

$$
\mathrm{d} \Omega=\mu A^{1 / 2} \mathrm{~d} \xi^{1} \mathrm{~d} \xi^{2} \mathrm{~d} \xi^{3} ; \quad A=\operatorname{det}\left(A_{i j}\right) .
$$

Equilibrium equations of shells

Starting from the three-dimensional principle of virtual work, and employing the kinematical approx-

† For convenience, we shall often use $\xi$ instead of $\xi^{3}$. imation eqn (2.1) introduced in the previous subsection, the equilibrium equations of the shell theory will be derived.

Denoting the first Piola-Kirchhoff stress by $T$, the pseudo-stress vector by $t$, the body force by $B$ and the deformation gradient by $\mathbf{F}$, the principle of virtual work for the three-dimensional solid shell of volume $\Omega$ and surface $\partial \Omega$ is

$$
\int_{\Omega} T \cdot \delta F d \Omega=\int_{\partial \Omega} t \cdot \delta 0 d \Lambda+\int_{\Omega} B \cdot \delta 0 d \Omega,
$$

in which $\partial \Omega=\partial \Omega_{+} U \partial \Omega_{-} U \partial \Omega_{n}$. The first Piola-Kirchhoff stress tensor $T$ can be written in terms of the pseudo-traction vector $\mathbf{T}^{\prime}$ in the following manner:

$$
\mathbf{T}=\mathbf{G}_{i} \mathbf{T} .
$$

Using eqn (2.3) the deformation gradient can be represented by

$$
\mathbf{F}=\boldsymbol{g}_{i} \mathbf{G}^{i},
$$

aiso $t$ is related to the first Piola-Kirchhoff stress as

$$
\mathbf{t}=\mathbf{N} \cdot \mathbf{T} \text { on } \partial \mathbf{\Omega},
$$

with $\mathbf{N}$ denoting the normal vector to the body surface $\partial \Omega$ in the initial configuration.

Substituting eqns (2.1) and (2.17) into (2.16), the internal virtual work becomes

$$
\begin{aligned}
& \int_{\Omega} \mathbf{T} \cdot \delta \mathbf{F} \mathrm{d} \Omega=\sum_{,=0}^{R} \int_{r} \\
& \times\left\{\mathbf{M}_{r}^{\mathbf{e}} \cdot \delta \mathbf{U}_{r, \boldsymbol{a}}+\mathrm{m}_{r} \cdot \delta \mathbf{U}_{r}\right\} \mathrm{d} \Gamma,
\end{aligned}
$$

where the quantities $\mathbf{M}_{r}^{a}$ and $\mathbf{m}_{r}$ introduced are referred to as generalized stress resultants. Explicitly, we have

$$
\mathbf{M}_{r}^{\alpha}=\int_{h_{1}}^{h_{2}} \mathbf{T}_{\boldsymbol{r}} f_{r} \mu \mathrm{d} \xi \quad \mathbf{m}_{r}=\int_{h_{1}}^{h_{2}} \mathbf{T}^{3} f_{r}^{\prime} \mu \mathrm{d} \xi .
$$

The component form of eqns (2.21) are given in Appendix A. Now, the surface traction in eqn (2.16) is to be expanded. Considering the top and bottom surfaces first, we find

$$
\int_{\varnothing \Omega_{+} U \Omega_{-}} \mathrm{t} \cdot \delta \mathrm{O} \mathrm{d} \Lambda=\sum_{r=0}^{R} \int_{r} q \cdot \delta U, \mathrm{~d} \Gamma,
$$

with

$$
\mathbf{q}=f_{r} \mathbf{t} W \mu \quad \text { at } \quad \xi=\left[h_{1}, h_{2}\right]
$$

and the scalar quantity $W$ is introduced to take into account the possibility of a variable thickness. Supposing that $h_{1}$ and $h_{2}$ denote the thickness variables function of $\xi^{\star}$ then 


$$
W=1+G^{\alpha \beta} \frac{\partial h_{1}}{\partial \xi^{\alpha}} \frac{\partial h_{2}}{\partial \xi^{\beta}}
$$

Now the integral that contributes to the tractions on the lateral surfaces is to be evaluated knowing that $N_{\alpha}$ and $N^{\alpha}$ are the covariant and the contravariant components respectively of the outward normal vector to the surface $\partial \Omega_{n}$. Clearly $N_{\alpha}$ is in general function of the parameters $\xi^{i}$. In addition, the outward normal vector to the boundary $\partial \Omega_{n}$ at $\xi=0$ is designated by $\bar{v}=\mathbf{N}\left(\xi^{1}, \xi^{2}, 0\right)$. Therefore, the surface term on $\partial \Omega_{n}$ can be written as

$$
\int_{\partial a_{n}} t \cdot \delta \mathrm{Ud} \boldsymbol{A}=\sum_{r=0}^{k} \int_{\partial r}\left(t_{r} \cdot \delta U_{r}\right) \mathrm{d} s,
$$

where

$$
t_{r}=\int_{h_{1}}^{h_{2}}\left(f_{r} t N^{\alpha} \bar{v}_{a} \mu\right) \mathrm{d} \xi \quad \bar{v}=\bar{v}^{\alpha} A_{\alpha}=\bar{v}_{\alpha} \mathbf{A}^{a} .
$$

Finally the body force term in the virtual work eqn (2.16) can be evaluated using similar procedures. Thus,

$$
\int_{\Omega} \mathbf{B} \cdot \delta \mathrm{Od} \Omega=\sum_{r=0}^{R} \int_{\Gamma}\left(\mathbf{B}_{r} \cdot \delta \mathbf{U}_{r}\right) \mathrm{d} r
$$

where

$$
\mathbf{B}_{s}=\int_{h_{1}}^{h_{2}}\left(f_{r} \mathbf{B}\right) \mu \mathrm{d} \xi
$$

It is now an easy task to determine the equilibrium equations and the boundary conditions. Making use of eqns (2.19)-(2.25) and taking into consideration the divergence theorem, we deduce the following equilibrium equations for the proposed shell theory:

$$
\left.\begin{array}{rlll}
\Delta \cdot \mathbf{M}_{r}+\mathbf{B}_{r}+\mathbf{q}_{r}=\mathbf{m}_{r} & \text { on } & \Gamma \\
\mathbf{M}_{r} \cdot \bar{v}=\mathbf{t}_{r} & \text { on } & \partial \Gamma_{2}
\end{array}\right\} 0 \leq r \leq R
$$

while $\Delta=A^{\alpha}\left(\partial / \partial \xi^{\alpha}\right)$. It is interesting to note that the latter equations are quite similar to the one derived previously by Naghdi [7]; however, eqns (2.26) are formulated on the basis of a full Lagrangean description.

\section{Constitutive relations for shells}

Our attention is subsequently turned to the derivation of the constitutive equations for the shell theory. At first, a general type of three-dimensional equation is given which can be utilized for a broad class of solids, namely, the class of incrementally linear materials; this class contains as special subcases: hyperelastic (or Green-elastic) and hypoelastic materials.

Thus, the three-dimensional constitutive relation for the incrementally linear material is

$$
\mathbf{T}=\mathbf{H}: \mathbf{F}
$$

in which the incremental moduli tensor $\mathbf{H}$ is a fourth* order tensor which, in general, depends on current stress and deformation state as well as internal variables. It is also noted that the analysis is based on an isothermal behavior.

For the two-dimensional constitutive laws of the shell theory, the increments of the stress resultants defined in eqn (2.21) become

$$
\mathbf{M}_{r}^{\alpha}=\int_{h_{l}}^{h_{2}} \mathbf{T} f_{r} \mu \mathrm{d} \xi \quad \mathbf{m}_{r}=\int_{h_{l}}^{h_{2}} \mathbf{T} f_{r} \mu \mathrm{d} \xi,
$$

which can be expanded in terms of the contravariant components of the first Piola-Kirchhoff stress tensor yielding, in view of eqns (2.27) and (2.28)

$$
\begin{aligned}
& \dot{M}_{p}^{\alpha_{i}}=\sum_{s=0}^{R}\left[\int_{h_{1}}^{h_{2}} f_{n} f_{s}\left(\mathbf{A}^{i} \mathbf{G}^{\alpha} \cdot \cdot \mathbf{H} \cdot \mathbf{G}^{\beta} \mathbf{A}\right) \mu \mathrm{d} \xi\right] \dot{\Theta}_{s, j \beta} \\
& +\sum_{\xi=0}^{R}\left[\int_{h_{1}}^{h_{2}} f_{f} f_{s}\left(\mathbf{A}^{i} \mathbf{G}^{\alpha_{*}} \cdot \mathbf{H} \cdot \mathbf{G}^{3} \mathbf{A}\right) \mu \mathrm{d} \xi\right] \Theta_{s \beta}
\end{aligned}
$$

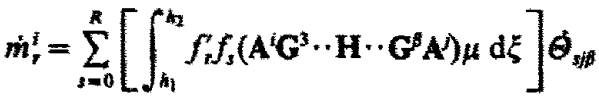

$$
\begin{aligned}
& +\sum_{j=0}^{R}\left[\int_{h_{1}}^{h_{2}} f_{k} f_{k}^{\prime}\left(\mathbf{A}^{i} \mathbf{G}^{3} \cdot \cdot \mathbf{H} \cdot \mathbf{G}^{3} \mathbf{A}\right) \boldsymbol{\mu} \mathrm{d} \xi\right] \boldsymbol{\theta}_{p j},
\end{aligned}
$$

in which the following notations are introduced

$$
\Theta_{\text {nix }}=A_{i} \cdot d_{t, *} \text { and } \Theta_{s \beta}=A_{i} \cdot d_{s} .
$$

The quantities $\Theta_{\text {tul }}$ are not the increments of any strain quantity. In a more compact form, the expressions in eqn (2.29) can be rewritten as

$$
\begin{gathered}
\dot{M}_{r}^{\alpha i}=\sum_{i=0}^{S} \mathscr{H}_{r s}^{a j j} \dot{\Theta}_{s j k} \\
\dot{m}_{r}^{i}=\sum_{i=0}^{R} \mathscr{H}_{r s}^{3 j i k} \dot{\Theta}_{s, k},
\end{gathered}
$$

where

$$
\mathscr{H}_{r t}^{i n k}=\int_{h_{k}}^{h_{2}} f_{r}^{(n)} f_{\xi}^{(n)}\left(\mathbf{A} \mathbf{G}^{i} \cdot \mathbf{H} \cdot \mathbf{G}^{i} \mathbf{A}^{k}\right) \mu \mathrm{d} \xi
$$

with (i) denoting the derivative with respect to for $i=3$ only. Now, the above components are established in a convenient and simplified form suitable for implementation in the finite element scheme. Indeed, the two-dimensional incremental moduli $\mathscr{W}_{r s}^{/ 4 t}$ are in dependent of the rate of the displacement vectors $\mathrm{U}_{z}$.

As to the fourth-order tensor of the threedimensional moduli $H$ entering in eqn (2.31), it is taken with respect to a mixed type of basis, namely: 


$$
\mathbf{H}=H^{i k k} \mathbf{G}_{i} \mathbf{g}_{j} \mathbf{g}_{k} \mathbf{G}_{l},
$$

using the results just obtained, eqns (2.31) become

$$
\mathscr{H}_{r s}^{i j k l}=\int_{h_{1}}^{h_{2}} f_{r}^{(n)} f_{s}^{(n)}\left(\mathbf{A}^{j} \cdot \mathbf{g}_{m}\right) H^{u m n l}\left(\mathbf{g}_{n} \cdot \mathbf{A}^{k}\right) \mu \mathrm{d} \xi .
$$

It is of interest to note that for the particular case of the existence of a strain energy function $W$ allows one to obtain

$$
\mathbf{M}_{r}^{\alpha}=\frac{\partial \Psi}{\partial \mathbf{d}_{r, z}} \quad \mathbf{m}_{\mathrm{r}}=\frac{\partial \Psi}{\partial \mathbf{d}_{r}} \quad \Psi=\int_{h_{1}}^{h_{2}} W \mu \mathrm{d} \xi ;
$$

expression $\Psi$ may simply be called the surface energy density of the shell.

In component form the constitutive eqns (2.34) become

$$
M_{r}^{\alpha i}=\frac{\partial \Psi}{\partial \Theta_{r i \alpha}} \quad m_{r}^{i}=\frac{\partial \Psi}{\partial \Theta_{r i 3}} .
$$

In addition, the two-dimensional incremental moduli given in eqn (2.33) can simply be rewritten explicitly in function of $\Psi$ as

$$
\mathscr{H}_{r s}^{p l k l}=\frac{\partial^{2} \Psi}{\partial \Theta_{r l j} \partial \Theta_{s k l}} .
$$

\section{Material models employed}

The existence of a strain energy density function $W$ per unit undeformed volume is considered for an isotropic material; thereby, we have

$$
W(\mathbf{F}) \equiv W(\mathbf{C}) \equiv W(\mathrm{I}, \mathrm{II}, J),
$$

in which $\boldsymbol{C}$ is the right Cauchy-Green strain tensor, viz.

$$
\mathbf{C}=\mathbf{F}^{T} \cdot \mathbf{F} .
$$

Also I and II are the first and second invariants of $\mathrm{C}$ and the invariant $J$ already given in eqn (2.7) can be defined in function of the third invariant III of C as

$$
J=\sqrt{ } \text { III. }
$$

Now, we propose two different types of hyperelastic materials. Indeed, for realistic behavior, two particular mathematical conditions are of interest: the energy growth condition, and the ellipticity conditions. An extensive development of the latter argument is reviewed by Knowles and Sternberg [13] in a series of papers.

The growth condition is a physically reasonable requirement given that it states the imposition of an infinite amount of energy to reduce a body volume to null. By requiring the material to be always elliptic, we avoid the occurrence of a shear band type of instability.

(a) The first model proposed has the following form:

$$
W=A\left(\mathrm{I}^{2}-4 \mathrm{II}\right)+B(\mathrm{I}-\mathrm{II})-B
$$

and

$$
\begin{array}{r}
A=\frac{1}{8} \bar{\mu} \frac{1-v}{1-2 v} \text { and } B=\frac{1}{4} \bar{\mu} \frac{1}{1-2 v} \\
\bar{\mu}=\frac{E}{2(1+v)},
\end{array}
$$

where $E$ is Young's modulus, $v$ is Poisson's ratio and $\bar{\mu}$ is the shear modulus. The quantities $A$ and $B$ are computed on the grounds that for small strains $\partial^{2} W / \partial \mathbf{E} \partial \mathbf{E}$ leads to the standard plane strain twodimensional elastic moduli defined within the context of linear theory. Obviously the growth condition is not met. This function is to be used for the analytical pure bending problem presented in the next section. It is also noted that the reason for employing this material is its analytical simplicity.

(b) The second strain energy function is

$$
W=A(-\operatorname{Ln} J+J-1)+B(I-2 J),
$$

where

$$
A=\bar{\lambda}+\bar{\mu} \quad B=\bar{\mu} / 2,
$$

where $\bar{\lambda}, \bar{\mu}$ are the Lamé constants of linear elasticity.

It can be shown that for small strains this material is a linearly elastic one with Lame constants $\bar{\lambda}$ and $\bar{\mu}$. It is also not difficult to see that for $J \rightarrow 0$ then $W \rightarrow \infty$. In addition, in the limit when $v$ approaches $1 / 2$ the Mooney Rivlin type of material behavior is exhibited. Employing the ellipticity condition, we find that the strain energy density in eqns $(2.42)-(2.43)$ meets the requirement of strong ellipticity [13]. Furthermore, it is noted that similar types of material were also presented by Ogden [14] and Oden [15].

\section{APPLICATIONS}

Our goal subsequently is to establish analytical and numerical comparisons between the shell theory outlined previously and exact theories using the simplest possible problems.

\section{Finite strain pure bending of a plate}

In the case of finite strain, we find that the simplest meaningful problem that can be solved analytically is the case of a plate in pure bending (see Ref. [16]). The position vector of a generic point of the deformed shell is described by

$$
\mathbf{p}=r+f_{1} \mathbf{d}_{1}+f_{2} \mathbf{d}_{2},
$$



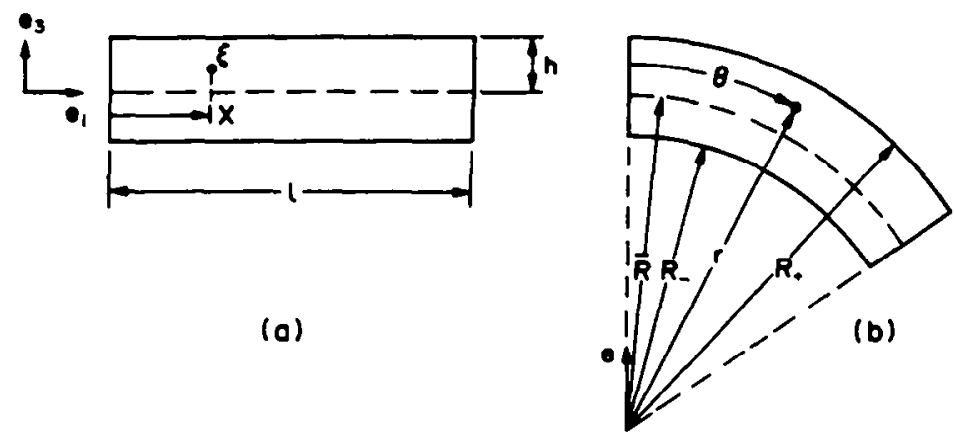

Fig. 2. (a) Undeformed plate and (b) deformed plate subjected to pure bending.

where $f_{1}$ and $f_{2}$ are the Legendre polynomials of order 1 and 2 respectively. The importance of the aforementioned theory relies on the absence of the contradiction that arises due to the Kirchhof-Love assumptions in shells. (Note that the exact pure bending problem using the same material is solved in Appendix B.) In the context of linear theories, it is worth mentioning that Hilderbrand et al. [17] proposed similar assumptions to the one in eqn (3.1).

In the undeformed configuration, the body $\Omega$ is a semi-infinite fat plate bounded by the planes $\xi^{\prime}=X=0, X=1$ and $\xi= \pm h$. The plate is then deformed into a portion of a cylinder bounded by curved surfaces $r(h)=R_{+}$and $r(-h)=R_{-}$as shown in Fig. 2; henceforth, the position vector in the deformed state is

$$
\mathrm{p}(X, \xi)=\mathrm{e}(X) r(\xi)
$$

where $e(X)$ represents the unit vector as

$$
\mathbf{e}(X)=\sin (\theta) \mathbf{e}_{1}+\cos (\theta) \mathbf{e}_{3} \quad \theta=\frac{X}{\bar{R}},
$$

with $k$ denoting the radius of curvature of the unstretched plate fibers at the current configuration.

In accordance with the kinematical assumption adopted in eqn (3.1), we have

$$
r(z)=R+f_{1}(z) d+f_{2}(z) b \quad z=\xi / h,
$$

where $R=d_{0}+b / 2$ and $R, d$ and $b$ denote the magnitudes of $d_{0}+d_{2} / 2, d_{1}$ and $d_{2}$ respectively. Also the radius of curvature $\bar{R}$ describes a surface for which fibers outside this surface are in extension and those inside are in compression at an instantaneous stage of deformation, whereas $R$ is merely the curvature at $\xi=z=0$.

The second Piola-Kirchhoff stresses for a hyperelastic material are given by the following relation:

$$
T^{i j}=2 \frac{\partial W}{\partial g_{i j}},
$$

where $W$ is defined in eqn (2.40) and in the case of a plane strain condition the two invariants are

$$
\mathrm{I}=g_{11}+g_{33} \quad \mathrm{II}=g_{11} g_{33}
$$

From eqns (3.2)-(3.6) the stress components become

$$
\begin{aligned}
& T^{11}=2\left\{2 A\left(g_{11}-g_{33}\right)+B\left(1-g_{33}\right)\right\} \\
& T^{33}=2\left\{2 A\left(g_{33}-g_{11}\right)+B\left(1-g_{11}\right)\right\} .
\end{aligned}
$$

Now, noting that the basis vectors are given by $A_{i}=e_{i}$, and using the expressions in eqns (A.1) and (A.2), the stress resultant components are obtained, viz.

$$
\begin{aligned}
M_{r}^{11} & =h \int_{-1}^{1} f_{r} T^{\prime \prime}\left(\mathbf{g}_{1} \cdot \mathbf{e}_{1}\right) \mathrm{d} z \\
M_{r}^{13} & \left.=h \int_{-1}^{1} f_{r} T^{11} \lg _{1} \cdot \mathbf{e}_{3}\right) \mathrm{d} z \\
m_{r}^{\prime} & =\int_{-1}^{1} f_{r}^{\prime} T^{33}\left(\mathbf{g}_{3} \cdot \mathbf{e}_{1}\right) \mathrm{d} z \\
m_{r}^{3} & =\int_{-1}^{1} f_{r}^{\prime} T^{33}\left(\mathbf{g}_{3} \cdot \mathbf{e}_{3}\right) \mathrm{d} z .
\end{aligned}
$$

Referring to eqn (A.4) and ignoring body forces and surface tractions the fundamental equations for the plane strain pure bending problem are

$$
\frac{\partial M_{r}^{11}}{\partial X}=m_{r}^{\prime} \quad \frac{\partial M_{r}^{13}}{\partial X}=m_{r}^{3}, r=0,1,2
$$

employing the relations in eqns (3.4)-(3.8) and substituting the results into eqn (3.9) the following three equilibrium equations are derived:

$$
\begin{aligned}
& \sum_{(, \mu, N)=0}^{2}\left\{2 A(\tilde{\kappa} \bar{h})^{4} \int_{-1}^{1} f_{r} f_{S} f_{u} f_{v} \mathrm{~d} z\right. \\
& -(2 A+B)(\bar{\kappa} \bar{h})^{2} \int_{-1}^{1}\left(f_{r} f f_{u}^{\prime} f_{v}^{\prime}+f_{r}^{\prime} f_{i}^{\prime} f_{v} f_{v}\right) \mathrm{d} z \\
& \left.+2 A \int_{-1}^{1} f_{,}^{\prime} f^{\prime} f_{u}^{\prime} f_{\mathrm{v}}^{\prime} \mathrm{d} z\right\} d_{1} d_{\mathrm{u}} d_{\mathrm{v}}
\end{aligned}
$$




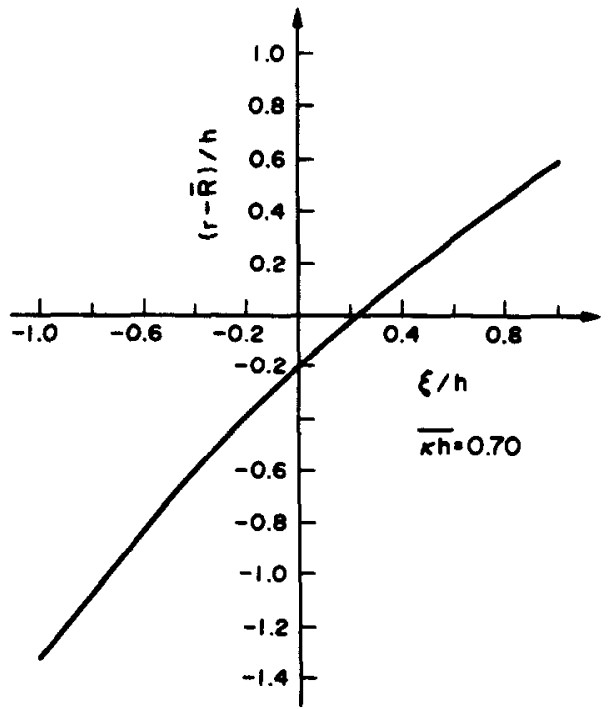

Fig. 3. Displacement vs thickness.

$$
\begin{aligned}
& +\sum_{i=0}^{2}\left\{B ( \overline { \kappa } h ) ^ { 2 } \left[(\bar{\kappa} h)^{2} \int_{-1}^{1} f_{r} f_{l} \mathrm{~d} z\right.\right. \\
& \left.\left.+\int_{-1}^{1} f_{i}^{\prime} f_{i}^{\prime} \mathrm{d} z\right]\right\} \quad d_{t}=0 \quad r=0,1,2,
\end{aligned}
$$

in which the following notations are introduced:

$$
\begin{array}{rll}
\bar{\kappa}=1 / \bar{R} \quad \kappa=1 / R \quad d_{0}=1 \quad d_{1}= & d / R \\
& d_{2}=b / R
\end{array}
$$

and where $A$ and $B$ are defined in eqn (2.41).

The displacement and stress distributions are obtained from the exact (see Appendix B) and approx-

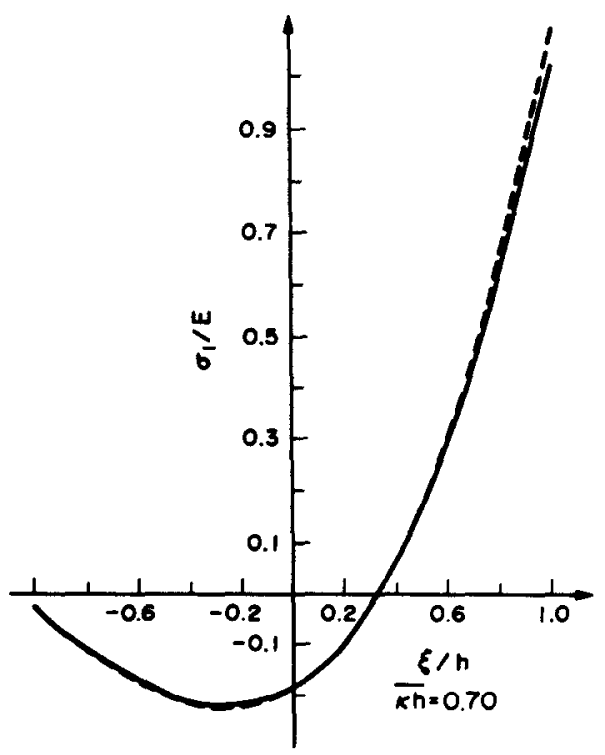

Fig. 4. Stress vs thickness. imate theories. The analysis was carried out with Poisson's ratio taken to be equal to 0.3 . The solution for the unknowns $R, d$ and $b$ (as function of $\bar{\kappa} \bar{h}$ ) was found using an incremental Newton-Raphson procedure.

Thereafter, displacements, axial stresses and bending moments were obtained for small curvatures and for large curvatures and large strains (see Figs 3-5).

For the small curvature case the agreements were very good $v i s-\grave{a}-v i s$ the classical theory leading to a maximum difference of the order $0\left(10^{-6}\right)$.

The non-dimensionalized distance of a material point from the unstretched fiber $[r(\xi)-\bar{R}] / h$ vs $z$ of the exact and approximate solutions are plotted in Fig. 3. The distributions are obtained for $\bar{\kappa} h=0.05$, 0.25 and 0.7 respectively. The results reveal good agreement to such an extent that the solutions look identical graphically at low curvatures. Even at very high curvature, $\bar{k} \bar{h}=0.7$ (Fig. 3), where strains are finite, the analytical outcome shows a percentage of error of $0.1 \%$ at the extreme fibers. The relevance of these results is very encouraging with regard to the kinematical assumption adopted here.

Also, the axial Cauchy stress distributions for the same curvature are plotted in Fig. 4 vs the nondimensionalized position variable $z=\xi / h$. At curvature $\bar{k} \bar{h}=0.05$ no difference is observed graphically and a maximum of $0.3 \%$ error is found from the exact solution. With increasing curvatures such as $\bar{\kappa} \bar{h}=0.25$ and 0.7 the difference in error percentage gradually increases. However, it is noted that even at fairly high curvature (i.e. $\bar{k} h=0.7$ ) the maximum error is about $1.1 \%$.

Finally, in Fig. 5 the plotted bending moments $M / E h^{2}$ vs the normalized curvature of the unstretched fibers reveal that the approximate theory (dashed lines) overestimates the exact one by a rather small amount. It is shown that a mere $2.8 \%$ discrepancy in the bending moment is noticed at high strain level where $\bar{\kappa} \bar{h}=0.7$.

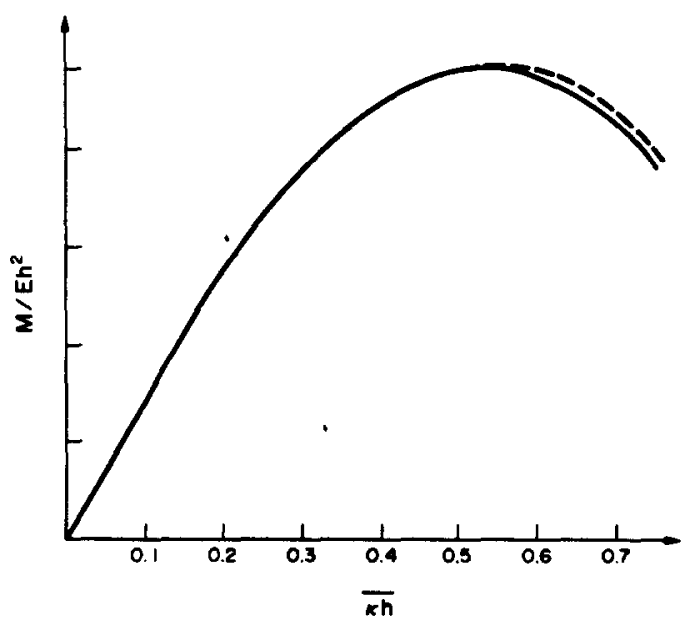

Fig. 5. Moment curvature relation for pure bending. 
However, it is noted that for Poisson's ratio greater than 0.45 the discrepancies between the exact and the approximate theories become larger. (It is thus noted that nearly incompressible materials are to be investigated in further work.)

Overall the results obtained with the shell theory in this section are consistent with the order of approximation utilized and represent adequately the deformation of the plates considered here in comparison with the exact solution given in Appendix B for the finite strain pure bending case.

As a next step, the finite element is utilized to solve more complex finite strain problems.

\section{Finite element formulation for shells}

Let us consider a shell or a plate embedded in a three-dimensional Euclidean space and bounded by the surfaces $\xi=h_{+}\left(\xi^{1}, \xi^{2}\right)$ and $\xi=h_{-}\left(\xi^{1}, \xi^{2}\right)$. Let the surface $\Gamma$ at $\xi=0$ of the body be spanned by the reference base vectors. Then the incremental form of the principle of virtual work in the absence of the lateral surface traction and in accordance to eqn (2.1) is

$$
\begin{aligned}
& \int \sum_{r=0}^{R}\left[\dot{M}_{r}^{\alpha_{r}} \delta \Theta_{r k}+\dot{m}_{r}^{i} \delta \Theta_{r 3}\right] \mathrm{d} \Gamma \\
& \quad=\int_{\Gamma} \sum_{r=0}^{R}\left[\dot{q}_{r}^{i} \delta \Theta_{r i 3}\right] \mathrm{d} \Gamma+\int_{\Gamma} \sum_{r=0}^{R}\left[\dot{B}_{r}^{i} \delta \Theta_{r i 3}\right] \mathrm{d} \Gamma,
\end{aligned}
$$

in which $\dot{M}_{r}^{\text {ai }}$ and $\dot{m}_{r}^{i}$ are the incremental stress resultants given in eqns (2.28) or (2.29), and $\dot{q}_{r}^{l}$ and $\vec{B}^{i}$ are the incremental assigned forces expressed in eqns (2.22) and (2.25) respectively.

The problems are solved iteratively employing a Newton-Raphson procedure. And to prevent the solution from deviating from the true equilibrium solution, correction terms are included on the righthand side of the just stated equation.

It is noted that the discretization procedure adopted is canonical and for a detailed description in the application to nonlinear continua one may refer to Oden's book [18], Zienkiewicz's [19] or to any standard finite element textbook.

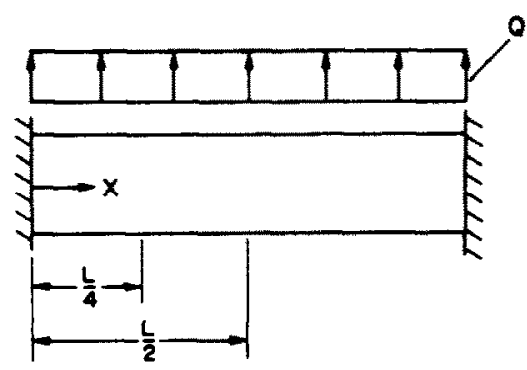

(a)
Results

The solutions of the problems considered subsequently are obtained by using on one hand the finite element method based on the shell equations aforementioned and on the other hand by applying the finite element procedure to the exact equations of elasticity.

Two-node elements were utilized to discretize the semi-infinite plate investigated based on the shell theory. And to compare the results, the exact finite element discretization is made utilizing bilinear elements. The finite element methods laid out in the present work are based on the isoparametric formulation.

For the hyperelastic material given in eqn (2.42), examples were solved, namely:

Case I. The clamped plate subjected to a uniform transverse load $g$ is investigated where the total length is taken to be first $L / h=10$ and then $L / h=40$ (Fig. 6).

Case II. The clamped plate is acted upon by a linearly distributed shear load and the length measures are the same as above.

In both cases Poisson's ratio is taken to be equal to $v=0.24$. It is noted that all quantities entering in the finite element solution process are nondimensionalized.

The kinematical terms $\mathrm{d}_{\text {, }}$, for $r=0,1,2$ and $r=0$, 1, 2, 3 are employed successively for the thick and thin plate problems.

Let us first investigate the thick plate for case I. It is noted first that all quantities plotted are nondimensionalized. In Fig. 7, the normal stresses $S^{11}$ are plotted at $X=L / 2.0$ for $r$ ranging from 0 to 2 and from 0 to 3 successively. We can see that for this particular thick structure the exact (solid texture) and the approximate (dashed texture) curves overlap fairly well. It is also important to realize that at $X=0.0$ the normal strain reaches a maximum level of $163 \%$. The curves for the stresses present a discrepancy of the order of $1.5 \%$ when the case for $r=0,1,2$ is considered, whereas for $r=0,3$, the differences are almost null. The latter observations

Fig. 6. Geometry and loading condition of the plate. (a) Uniformly distributed transverse load and (b) linearly distributed shear load. 

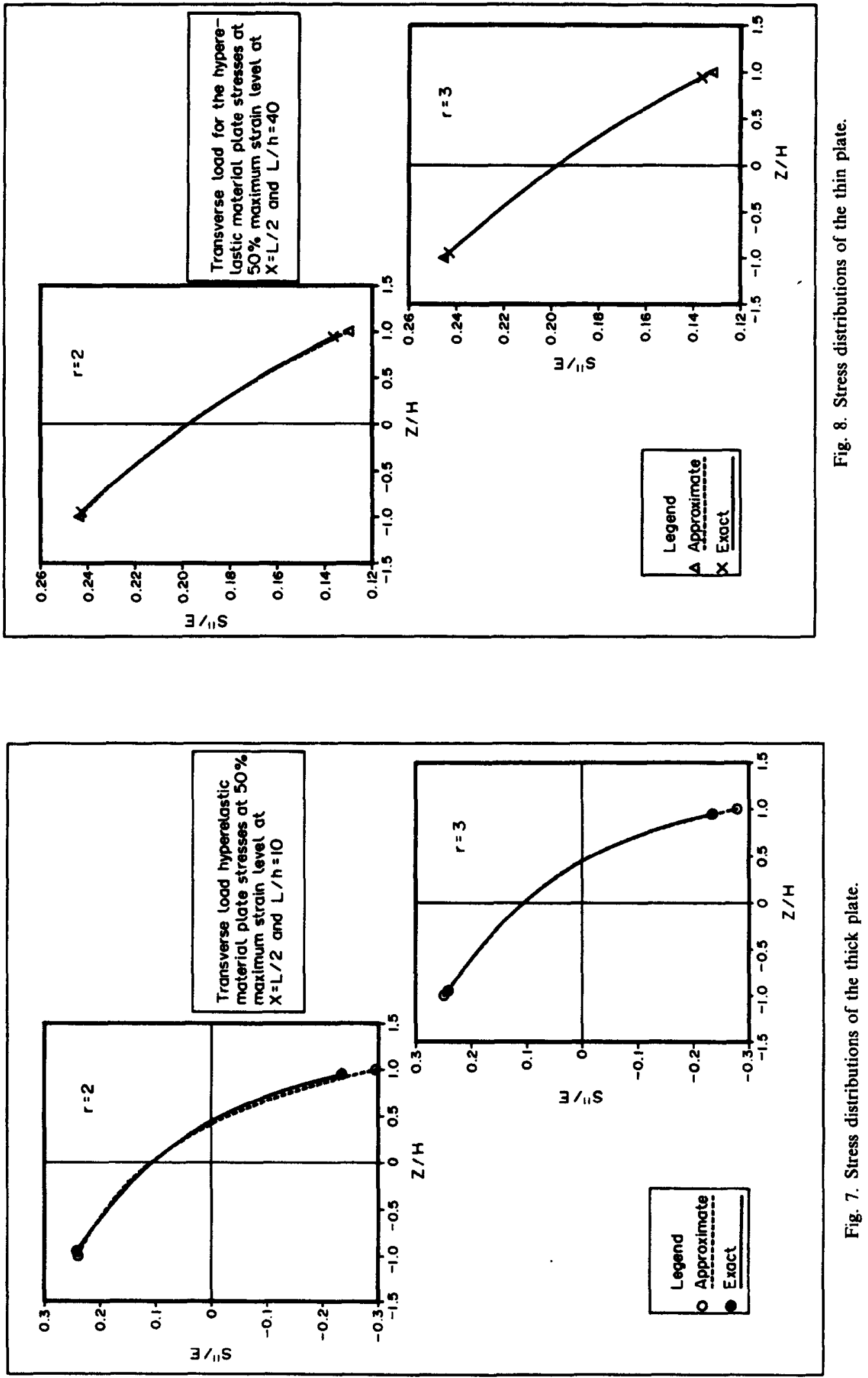


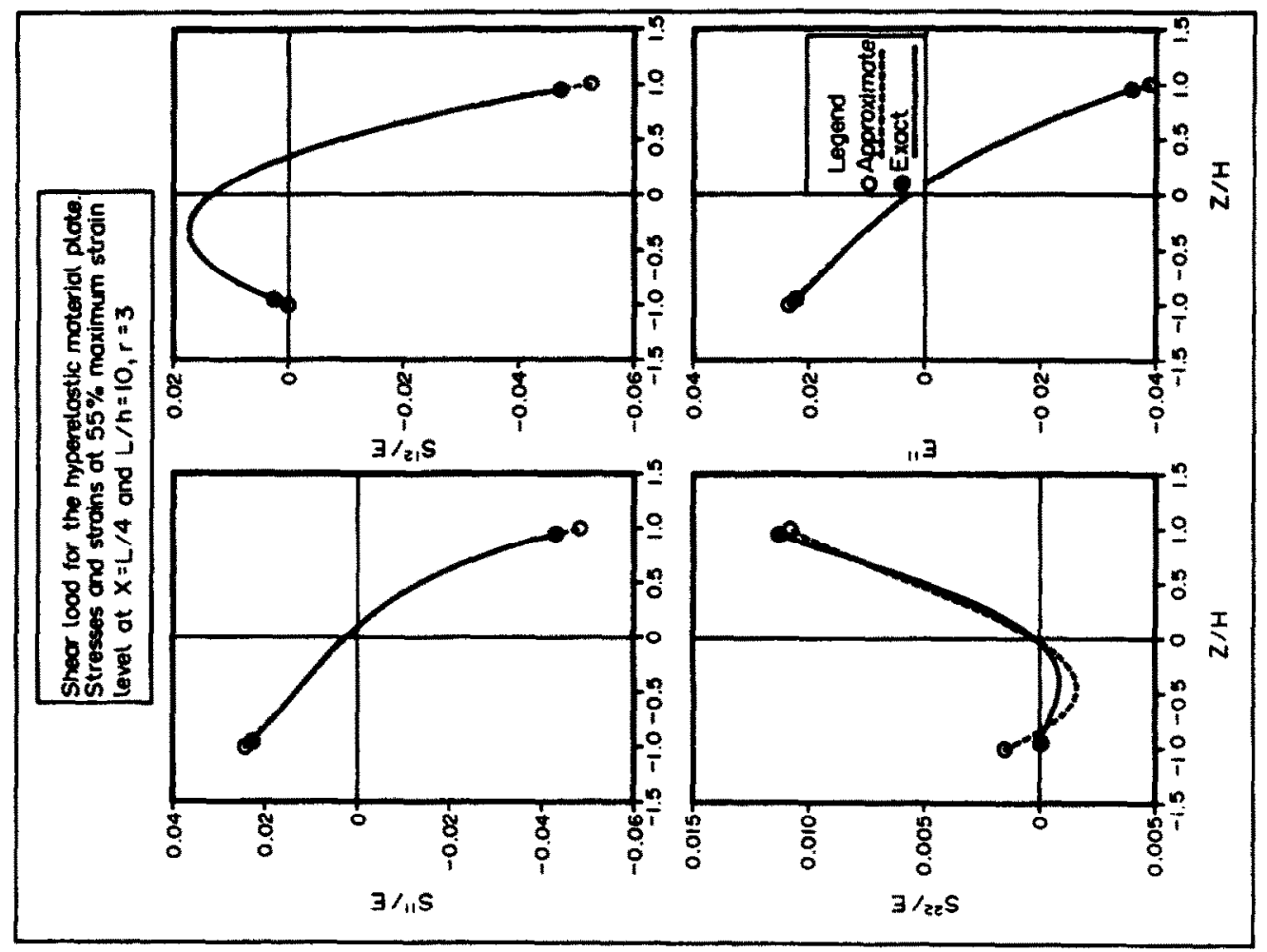

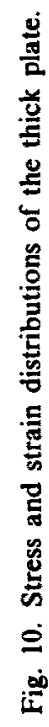

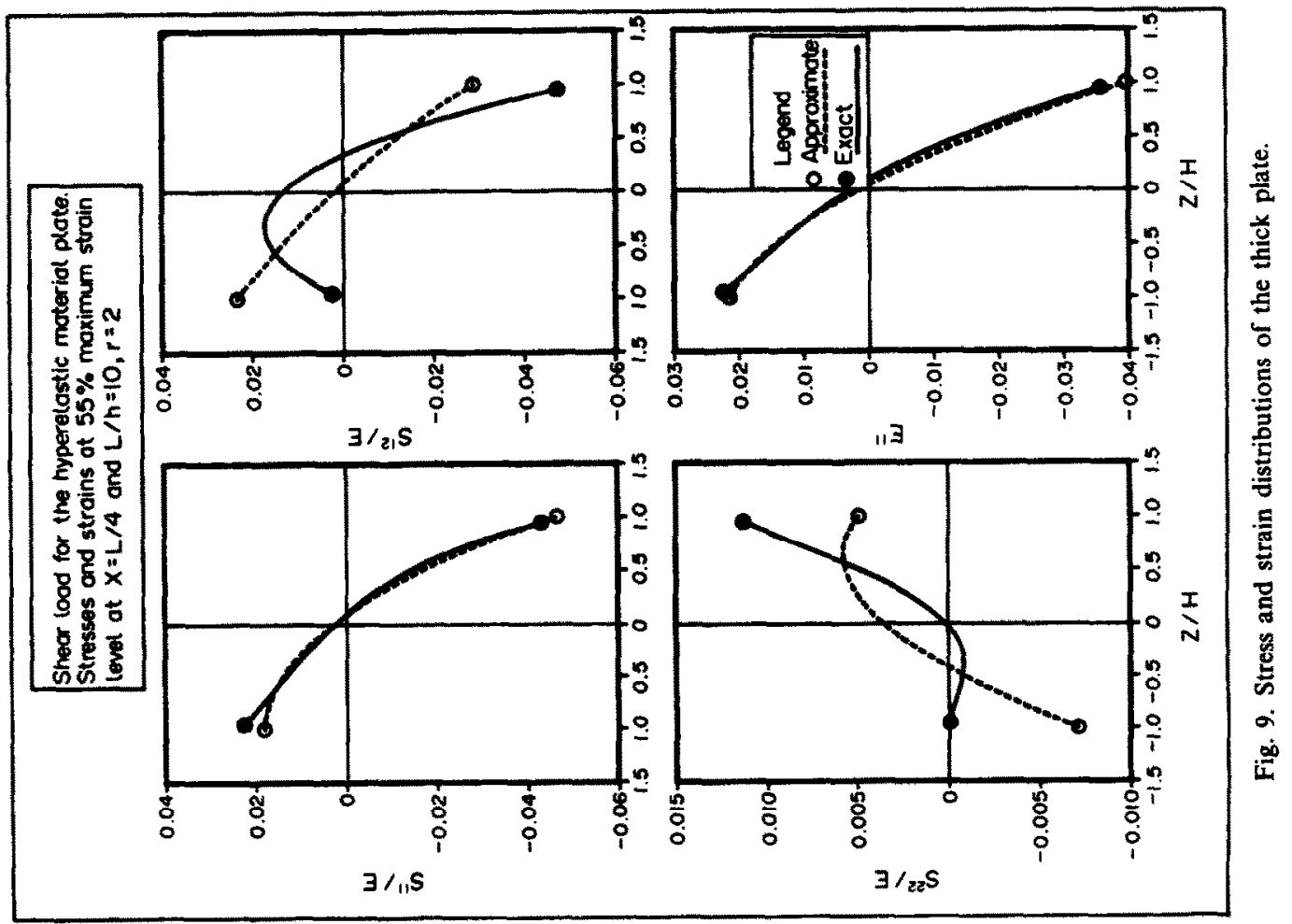


show that the series in (2.1) converges quite rapidly. Noteworthy is the fact that the stresses and the strains of the same case and same properties present similar accuracy.

Also for the thin case where $L / H=40$, we have recorded the results in Fig. 8 where the maximum strain level is $50 \%$ at mid-section. The differences between the exact and the approximate solutions are merely noted to be reduced to almost null. It is of great significance to note that the strains at the wall attain a maximum level of $250 \%$. Although the just mentioned value represents an extremely high strain level, the results are surprisingly good and consistent with the kinematical assumption adopted in the present work.

Finite strain and large deformation problems are also analyzed for case II. Shear effect in finite analyses is important, as, for example, rigid punch problems often require a careful consideration of shearing deformations. Thus, the plate acted upon by a shear load is investigated and the results are recorded for the case where $L / H=10$ and $r=0,1,2$ and $r=0$, $1,2,3$ respectively in plots 9 and 10 .

The normal stress and strain in the 1-direction exhibit a maximum of $3.5 \%$ error when $R=2$. However, for the higher order case the discrepancy is negligibly small. Now, the approximate shear stress $S^{12}$ curve does show a bad comparison with the exact one; this is also true for the normal stress in the $\xi$-direction. The latter results are partly due to the fact that the stresses' quantities in the thickness direction involve dominantly the metric $g_{22}$. When the higher-order terms (i.e. $r=0,1,2,3$ or $R=3$ ) are considered, the stresses show results that are considerably improved. And of particular importance is the behavior of the shear stress curves in Fig. 10, which show adequate agreement. The latter observations again show a surprisingly fast convergence behavior of the series in eqn (2.1).

For the thin plate, we note that the stresses $S^{12}$ and $S^{22}$ exhibit better results than previously noted for the thick plate when $r=0,1,2$. It is interesting to note that the shear stress $S^{12}$ and the normal one $S^{22}$ are nonlinear. This is of significant interest given that the classical theories neglect the latter quantities. Even for thin plates we can ascertain from the above observations that normal and shear stresses in the $\xi$-direction cannot be ignored by fear of leading to significant discrepancies.

For the sake of obtaining more physical insight, the undeformed and deformed shapes of the various plates are presented subsequently in Figs 11-14 (half of the plate is considered).

Case I is presented in Fig. 11 for the thick plate, and in Fig. 12 for the thin case. It is clear in the subsequent figures that fibers originally normal to the undeformed plate middle surface are neither normal nor straight in the deformed configurations. The latter observations are especially noticeable at the clamped end where the strain level is of the order of
$163 \%$. Interestingly, we see that the cross-sectional fibers are deformed nonlinearly.

It is also of particular relevance to note that even at one fiber away (see figures) from the wall the slope is not zero as would be the case if Kirchhoff's assumptions were utilized. Now, the discrepancies are rather minor for the thick and the thin cases. It is worth noting that for case I, in the thin case, the transverse displacements at midspan are as high as approximately 11 times the intial total thickness and for case II (Fig. 14) the transverse displacements are 5 times $2 H$.

Thus, for such high strain and displacement magnitudes the results are overall fairly encouraging. Indeed, the non-normality condition observed in the figures conforms to a realistic plate model undergoing large deformations.

\section{CONCLUSION}

Based on only one consistent high-order kinematical approximation a nonlinear shell theory is presented and facilitates incrementally linear constitutive relations including material and geometrical nonlinearity. Of special interest in this paper are applications using hyperelastic materials exhibiting finite strains and large deformations.

To assess the accuracy of the theory, analytical and numerical applications within the nonlinear context are studied. A simple finite pure bending problem is solved analytically using this approximate theory and is compared with the exact solution. The results show that good agreements exist between the theories even at fairly high curvatures.

Also, the finite element method is employed to solve more complex problems in which shear and normal effects are important and in particular two cases are considered: a semi-infinite plate subjected respectively to a uniformly distributed transverse load and a linearly varying shear load at the top surface. An exact finite element analysis is employed to compare our results via the theory proposed. The outcome reveals that the stresses and the strains agree quite well especially for the quantities in the plane of the plate. As to the shear and the normal stresses in the thickness direction, some discrepancies arise and are reduced significantly when the parameter $\boldsymbol{R}$ increases from 2 to 3 . In the latter case the differences for the stresses are almost null when the thin plate is considered even at high strain.

The displacements are plotted and show that in all cases the approximate theory compares adequately with the exact theory. The deformed shapes of the plates reveal clearly that the cross-sectional fibers are not straight and hence emphasize the importance of the higher-order terms in the kinematics.

All in all the results are very encouraging; nevertheless, additional investigation will prove quite useful. 

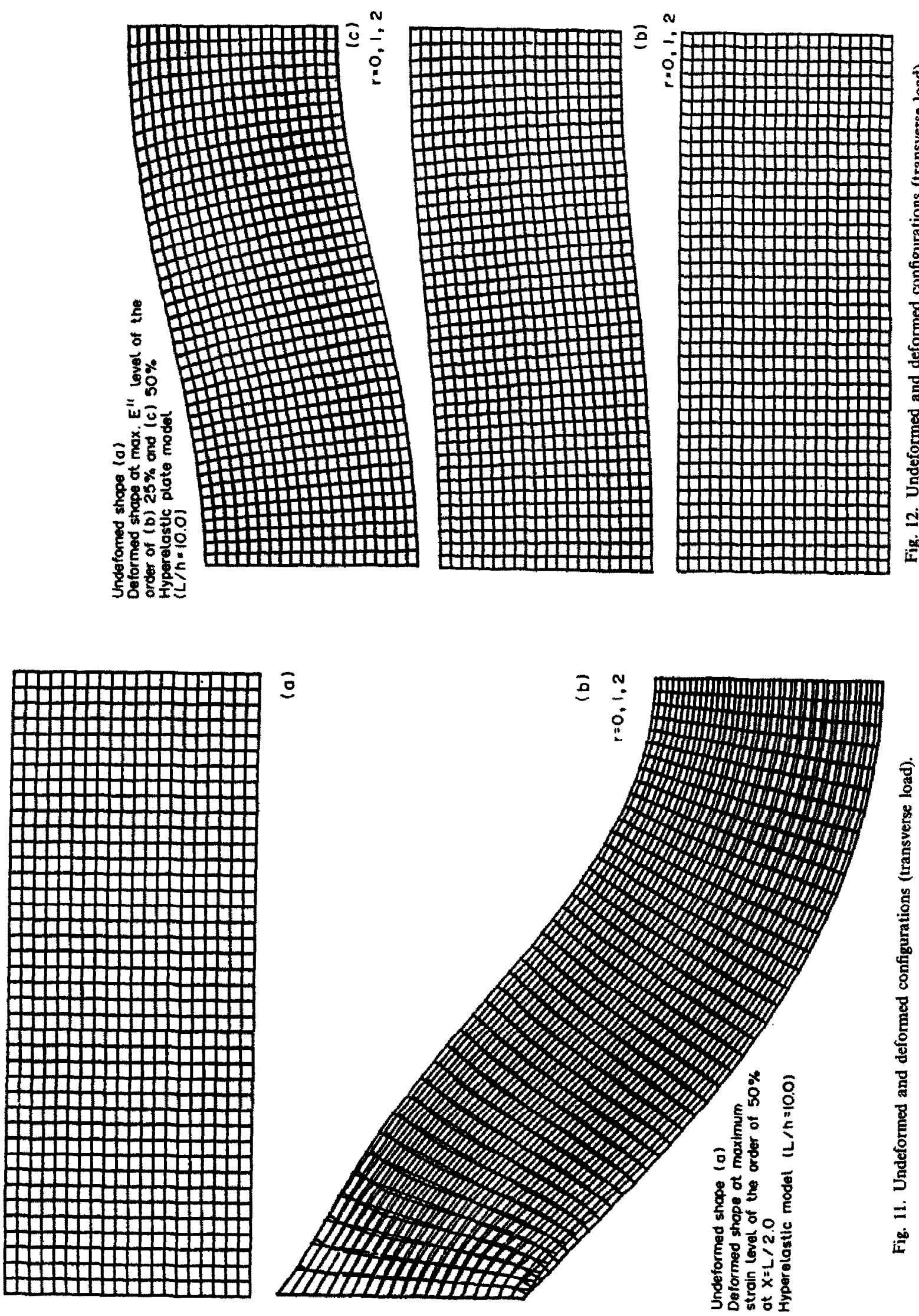

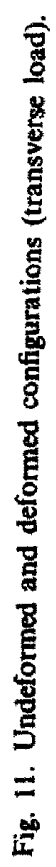



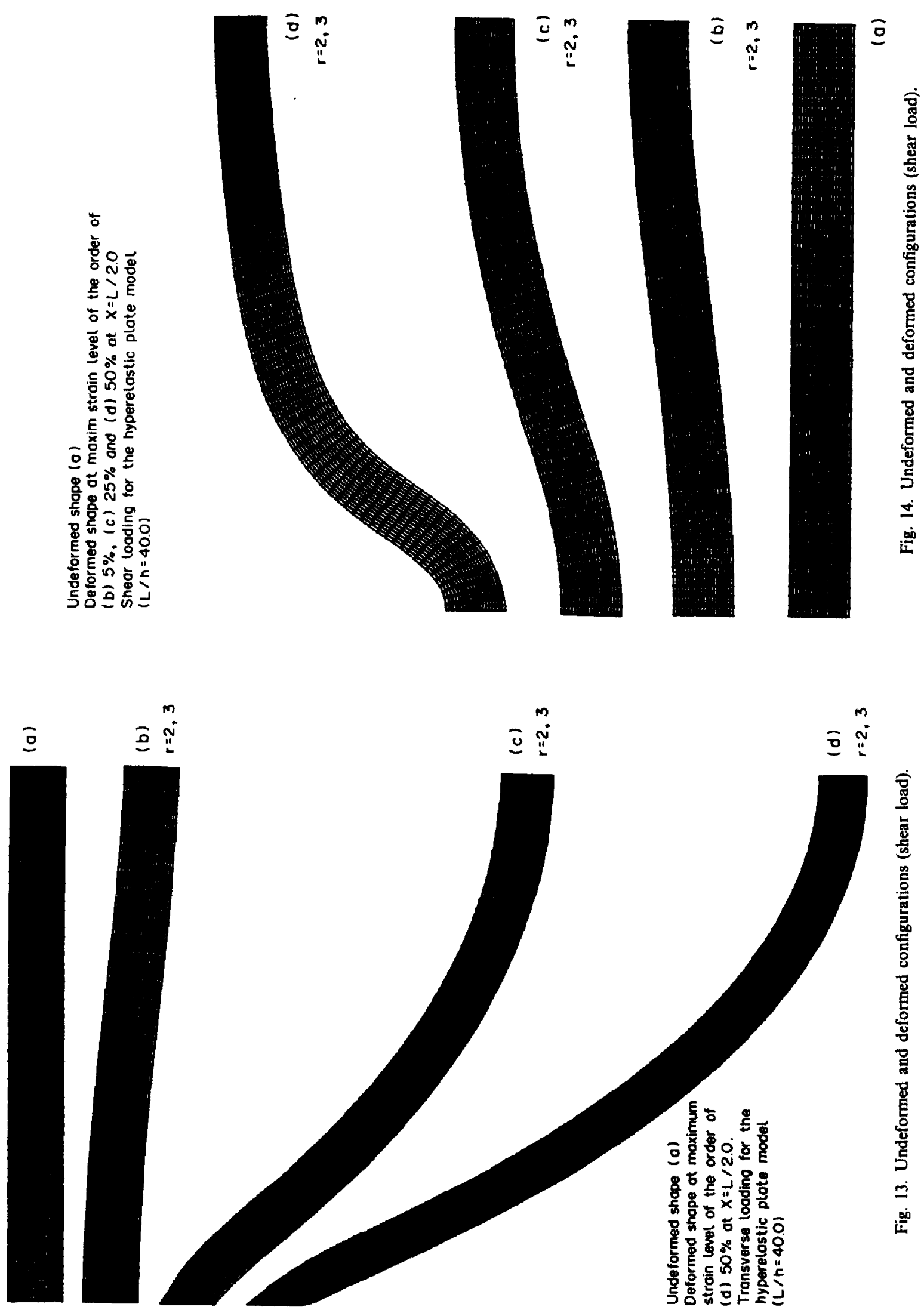

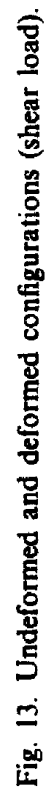


Acknowledgements - The author wishes to extend his sincere thanks to his advisor Prof. N. Triantafyllidis for his help during the course of this work. I also wish to thank Prof. N. Kikuchi (Applied Mechanics and Mechanical Engineering Department, The University of Michigan) for his helpful comments on the finite element method. This work was supported by a grant by TRW. The financial support of the Aerospace Engineering Department, The Unjversity of Michigan is also greatly approciated.

\section{REFERENCES}

1. A. E. Love, A Treatise on the Mathematical Theory of Elasticity, 4th Edn. Dover, New York (1944).

2. E. Reissner, The effect of transverse shear deformation on the bending of elastic plates. J. appl. Mech., Trans. ASME 67, 69 (1945).

3. R. D. Mindlin, Influence of rotatory inertia and shear on flexural motions of isotropic elastic plates. $J$. appl. Mech. 18, 31-38 (1951).

4. J. L. Sanders, Nonlinear theories for thin shells. $Q$. appl. Math. 21, 21-36 (1963)

5. W. T. Koiter, On the nonlinear theory of thin elastic shells. Proc. Kon. Ned. Akad. Wet. Ser. B69, 1-54 (1965).

6. B. Budiansky, Notes of nonlinear shell theory, J. appl. Mech. 35, 393-401 (1968).

7. P. M. Naghdi, Theory of plates and shells. In Handbuch der Physics, Vol. VI, a/2. Springer, Berlin (1971).

8. Y. Yokoo and $H$. Matsunaga, A general nonlinear theory of elastic shells. Int. J. Solids Siruct. 10, 261-274 (1974).

9. S. J. Antman, Ordinary differential equations of nonlinear elasticity. Archs Rat. Mech. Anal. 61, 307-393 (1976).

10. T. J. R. Hughes and W. K. Liu, Nonlinear finite element analysis of shells: Part $\mathrm{I}$. Three-dimensional shells. Comput. Meths appl. Mech. Engng 26, 331-362 (1981).

11. H. Parish, Large displacements of shells including material nonlinearities. Comput. Meths appl. Mech. Engng 27, 183-214 (1980)

12. A. E. Green and W. Zerna, Theoretical Elasticity. Clarendon Press, Oxford (1954).

13. J. K. Knowles and E. Sternberg, On the failure of ellipticity of the equations for finite elastostatic plane strain. Archs Rat. Mech. Anal. 63, 321-336 (1977).

14. R. W. Ogden, Large deformation isotropic elasticity: on the correlation of theory and experiment for compressible rubberlike solids. Proc. R. Soc. Lond. A 328, 567-583 (1972).

15. J. T. Oden, Finite element methods for a class of nonlinear boundary-value problems in finite elasticity. In The Mathematics of Finite Elements and Applications III, (Edited by Mapelap), pp. 327-339. Academic Press, London (1979).

16. R. S. Rivlin, Large elastic deformations of isotrotropic materials VI. Phil. Trans. R. Soc. Lond. A 242, 173-195 (1950).

17. F. B. Hilderbrand, E. Reissner and G. R. Thomas, Notes on the foundations of the theory of small displacements of orthotropic shells. NACA-TN 1833 (1949)

18. J. T. Oden, Finite Element of Nonlinear Continua. McGraw-Hill, New York (1972)

19. O. C. Zienkiewicz, The Finite Element Method, 3rd Edn MoGraw-Hill, London (1977).

\section{APPENDIX A}

Component form of the stress resultants, loads and equilibritum equations

The component form of the stress resultants eqn (2.21) are obtained by noting that $\mathbf{M}_{r}^{\star}$ and $\mathbf{m}_{r}$ are quantities defined with respect to the reference surface bases, thus

$$
M_{r}^{\alpha i}=\int_{-h}^{+h} f_{r} \mathbf{T}^{\mu} \cdot \mathbf{A}^{i} \mu \mathrm{d} \xi \quad m_{r}^{i}=\int_{-h}^{+h} f_{r} \mathbf{T}^{3} \cdot \mathbf{A}^{i} \mu \mathrm{d} \xi,
$$

in which the pseudo-traction vector $\mathbf{T}^{i}$ can be written in terms of the contravariant components of the first Piola-Kirchhoff stress defined as

$$
\mathbf{T}^{i}=T^{i j} \mathbf{g}_{j} \text {. }
$$

Taking into account eqns (2.22), (2.24) and (2.25) the component forms of the surface loads, traction vectors and body forces defined in $\Gamma$ become

$$
\begin{aligned}
q_{r}^{i} & =f,\left.\left(t \cdot A^{\prime}\right) W \mu\right|_{z= \pm \hbar} \\
t_{r}^{i} & =\int_{-\hbar}^{+\hbar}\left(t^{\prime} \cdot A^{\prime}\right) N^{\alpha} v_{\alpha} \mu \mathrm{d} \xi \\
B_{r}^{i} & =\int_{-h}^{+\hbar} f_{r}\left(\mathbf{B}^{\prime} \cdot \mathbf{A}^{\prime}\right) \mu \mathrm{d} \xi .
\end{aligned}
$$

The equilibrium equations for shells and plates in component form can be derived by utilizing eqns (A.1), (A.3) and (2.26), namely:

$$
\begin{aligned}
& \left.M_{r}^{* \beta}\right|_{\alpha}-M_{r}^{\alpha 3} S_{\alpha}^{\beta}+B_{r}^{\beta}+q_{r}^{\beta}=m_{r}^{\beta} \\
& \left.M_{r}^{a 3}\right|_{\alpha}+M_{r}^{\alpha \beta} A_{\alpha \beta}+B_{r}^{3}+q_{r}^{3}=m_{r}^{3}
\end{aligned} \text { on } r
$$

and the index $r$ ranges from 0 to $R$, where $R$ is finite

In the above equations, a vertical stroke (|) implies covariant differentiation with respect to the reference surface metric $A_{\alpha \beta}$. It is also recalled that the mixed form expressions $\mathfrak{A}_{\beta}^{\alpha}$ are given by the relation in eqn (2.11). Now, at the boundaries the second of eqns (2.26) may be specified in component form as

$$
M_{r}^{\alpha \beta} v_{\alpha}=t_{r}^{\beta} \quad M_{r}^{\alpha 3} v_{\alpha}=t_{r}^{3} \text { on } \partial r .
$$

Substituting (A.1) -(A.3) into (A.4), one obtains a set of differential equations for plates and shells with $d$ as unknowns. The solutions may be obtained by applying the boundary conditions eqn (A.5). It is important to realize that eqns (A.4) and (A.S) are exact within the context of the present shell theory.

\section{APPENDIX B}

\section{Exact analytical solution of the pure plate bending}

In order to compare our results obtained analytically via the shell theory to the plane strain pure bending plate problem exposed previously, the exact solutions to the same problem are presented subsequently. Plane polar co. ordinates (i.e. $\xi^{\prime}=\Theta$ and $\xi^{3}=r$ ) are adopted subsequently. It is clear that theoretically in pure bending problems no shearing modes are present; therefore, the principal stresses act in the radial and circumferential directions. Let the stretch ratio $\lambda_{1}$ and $\lambda_{3}$ be defined as

$$
\lambda_{1}=\bar{\kappa} r \quad \lambda_{3}=\frac{\partial r}{\partial \xi}=r^{\prime},
$$

with $\bar{\kappa}$ given in eqn (3.10) and $r=r(\xi)$. Note that $1 / \bar{k}$ denotes the radius of curvature of the currently unstretched fibers.

In view of the principle stretch ratio given in eqn (B.1) the invariants defined in eqn (3.6) for a plane strain case become

$$
\mathbf{I}=\lambda_{1}^{2}+\lambda_{3}^{2} \quad \mathbf{I I}=\lambda_{1}^{2} \lambda_{3}^{2}
$$

and the principal Cauchy stresses are 


$$
\sigma_{\alpha}=\frac{\lambda_{a}}{\lambda_{1} \lambda_{3}} \frac{\partial W}{\partial \lambda_{a}}
$$

Hence, using the expressions given in eqns (B.2) and (B.3), the principal stresses are found to be

$$
\begin{aligned}
& \sigma_{1}=2 \frac{\lambda_{1}}{\lambda_{3}}\left[2 A\left(\lambda_{1}^{2}-\lambda_{3}^{2}\right)+B\left(1-\lambda_{3}^{2}\right)\right] \\
& \sigma_{3}=2 \frac{\lambda_{3}}{\lambda_{1}}\left[2 A\left(\lambda_{3}^{2}-\lambda_{1}^{2}\right)+B\left(1-\lambda_{1}^{2}\right)\right],
\end{aligned}
$$

and the nontrivial equilibrium equation is recorded as

$$
\sigma_{3}^{\prime}+\frac{r^{\prime}}{r}\left(\sigma_{3}-\sigma_{1}\right)=0
$$

in which the chain rule was employed.

Substituting eqn (B.1) into (B.4) and the results in (B.5), the following differential equation is obtained:

$$
\begin{aligned}
\left\{3 A\left(r^{\prime}\right)^{4}+\left(r^{\prime}\right)^{2}\left[B-(\bar{\kappa} r)^{2}(2 A+b)\right]\right. & \left.-(\bar{\kappa} r)^{2}\left[A(\bar{\kappa} r)^{2}+B\right]\right\}^{\prime}=0,
\end{aligned}
$$

and the boundary conditions lead to

$$
\sigma_{3}=0 \text { at } \xi= \pm h
$$

or using eqns (B.1) and (B.4) ${ }_{2}$, we arrive at

$$
\begin{aligned}
& 2 A\left[\left(r^{\prime}\right)_{-}\right]^{2}=(2 A+B)\left(\bar{\kappa} R_{-}\right)^{2}-B \\
& 2 A\left[\left(r^{\prime}\right)_{+}\right]^{2}=(2 A+B)\left(\bar{\kappa} R_{+}\right)^{2}-B
\end{aligned}
$$

at $\xi=-h$ and $\xi=+h$ respectively.
Employing eqns (B.6), (B.7), (2.40) and (2.41) the exact solution of the pure plate bending problem may be obtained, namely:

$$
\begin{aligned}
\bar{\kappa}(\xi+h) & =\frac{1}{2} \int_{-y}^{y}\{f(y, \mu)\}^{-1 / 2} \mathrm{~d} y \\
y & =(\bar{\kappa} r)^{2}-1, \quad-\mu \leq y \leq \mu,
\end{aligned}
$$

where the functional $f$ is defined by

$$
\begin{aligned}
& f(y, \mu)=(y+1)\{2 A(y+1)+B y \\
& \left.+\left\{4[2 A(y+1)+B y]^{2}-3 B(4 A+B)\left(y^{2}-\mu^{2}\right)\right\}^{1 / 2}\right\} / 6 A
\end{aligned}
$$

and $\mu$ is evaluated from

$$
2 \bar{k} h=\frac{1}{2} \int_{-\mu}^{\mu}[f(y, \mu)]^{-1 / 2} \mathrm{~d} y .
$$

The result just mentioned constitutes a nonlinear equation in the parameter $\mu$. A simple bisection method was utilized to solve the unknown. However, to evaluate the integral, the interval $(-\mu, \mu)$ was subdivided into 40 subintervals and a four-point Gauss-Legendre quadrature was used to calculate each subinterval integral. Within a few iterations convergency was achieved.

Finally, the exact bending moment is given as

$$
M=\int_{R_{-}}^{R_{+}} \sigma_{1}(r-\bar{R}) \mathrm{d} r,
$$

in which we recall that $\boldsymbol{R}$ is defined as the current radius of curvature of the current unstretched fibers of the plate. 